\title{
Hydrogen content in Kazarian galaxies
}

\author{
V.S. Tamazian \\ Astronomical Observatory "Ramon Maria Aller", University of Santiago de Compostela, P.O. Box 197, Santiago de Compostela, \\ Spain \\ e-mail: oatamaz@usc.es
}

Received November 5; accepted August 6, 1999

\begin{abstract}
Several H I related astrophysical parameters such as neutral hydrogen total mass and hydrogen mean projected density, indicative total mass as well as usual ratios $M_{\mathrm{H}} / L_{0}, M_{\mathrm{H}} / M_{\mathrm{i}}$ and $M_{\mathrm{i}} / L_{0}$ for 23 galaxies from Kazarian lists are derived. Their variation ranges are rather wide almost coinciding with those for normal galaxies belonging to the same morphological types. Kazarian galaxies do not constitute a homogeneous sample by any of the commonly used $\mathrm{H}$ I related parameters. Comparison with Markarian and blue Haro galaxies demonstrate that Kazarian galaxies have quite similar H I properties and contain rather high relative numbers $(\approx 30 \%)$ of peculiar objects. Brief comments on the peculiar galaxies Kaz 63, Kaz 65, Kaz 69 and Kaz 211 are given.
\end{abstract}

Key words: UV-excess galaxies — Kazarian galaxies hydrogen content

\section{Introduction}

The neutral hydrogen content is one of the most important global parameters of the galaxy, giving rise to a set of certain characteristics such as total H I mass and its projected mean density, H I mass to luminosity ratio etc. serving as a useful tools to investigate the physical conditions and evolutionary trends in galaxies.

Much work has been done to study H I content in the normal galaxies covering practically all morphological types and using large, statistically significant homogeneous samples.

These investigations have shown certain correlations between morphological type, mass, luminosity and total H I mass of the galaxy (Gouguenheim 1969; Roberts 1969; Balkowski 1973; Shostak 1978; Bottinelli et al. 1982; Roberts \& Haynes 1994; Solanes et al. 1996).

In particular, an extensive study of the $\mathrm{H} I$ integral properties of a large number of spiral galaxies has shown well defined continuous variation of $\mathrm{H}$ I parameters in an unbiased sample of galaxies ranging from Sa to the late spirals (Bottinelli et al. 1982). The latter article also provides carefully determined useful mean values of $\mathrm{H}$ I related parameters thus providing an important reference for further comparison with peculiar objects.

Obviously, a better knowledge of the integral properties of normal galaxies is mandatory when trying to find out and investigate peculiar objects as well as determine their peculiarity and specific properties.

A number of papers dedicated to the H I study in galaxies from specific lists such as Markarian (Bottinelli et al. 1973a, 1975), several compact (Carozzi et al. 1974), Haro (Bottinelli et al. 1973b) and Kiso survey galaxies (Maehara et al. 1988) based on observations made with Nançay radiotelescope have been published. They give valuable criteria for the classification and better understanding of the physical properties of these galaxies as well as demonstrate their certain peculiarities.

For example, it was found that non-Seyfert Markarian galaxies show a wide variety of intrinsic physical properties which are the same as those for normal galaxies (Bottinelli et al. 1973a). In this subclass of Markarian galaxies, however the objects appear to be overluminous for their morphological type (Bottinelli et al. 1975).

In the previous paper (Tamazian et al. 1997, hereafter Paper I) the hydrogen $21 \mathrm{~cm}$ line observational data obtained for the first time for 39 galaxies taken from Kazarian lists (Kazarian 1979; Kazarian \& Kazarian 1982) have been presented out of which 30 objects have shown the positive hint of detection. These observations were made with the Nançay radiotelescope in the course of several observing runs in 1996.

In the present article several global parameters such as H I mass, indicative total mass, mixed mean H I projected density, H I mass-to-luminosity and the other commonly used ratios derived from the observational data for 23 galaxies are given. Seven galaxies have been discarded either because of poor and uncertain detection with small 
Table 1. Main data for Kazarian galaxies

\begin{tabular}{|c|c|c|c|c|c|c|c|c|c|c|c|c|c|c|}
\hline No. & Type & $B(0)$ & $D$ & $a_{25}$ & $A_{25}$ & $L$ & $M_{\mathrm{H}}$ & $\sigma_{\mathrm{h}}$ & $M_{\mathrm{i}}$ & $\sigma_{\mathrm{i}}$ & $M_{\mathrm{H}} / L$ & $M_{\mathrm{i}} / L$ & $M_{\mathrm{H}} / M_{\mathrm{i}}$ & $\sigma_{\mathrm{h}} / A_{25}$ \\
\hline 1 & 2 & 3 & 4 & 5 & 6 & 7 & 8 & 9 & 10 & 11 & 12 & 13 & 14 & 15 \\
\hline 1 & $\mathrm{Sbc}$ & 13.06 & 47.3 & 1.35 & 18.6 & 19.5 & 6.3 & 4.81 & 54.7 & 41.9 & 0.32 & 2.81 & 11.5 & 25.9 \\
\hline 3 & $\mathrm{Sc}$ & 14.40 & 80.3 & 0.78 & 18.2 & 16.4 & 4.9 & 3.54 & 11.8 & 9.0 & 0.30 & 0.72 & 41.5 & 19.5 \\
\hline 18 & $\mathrm{SBbc}$ & 13.20 & 66.3 & 1.15 & 22.1 & 33.7 & 4.3 & 2.27 & 91.7 & 47.4 & 0.13 & 2.72 & 4.7 & 10.3 \\
\hline 19 & $\mathrm{SBbc}$ & 12.77 & 65.7 & 1.48 & 28.2 & 49.1 & 10.1 & 3.31 & 131.3 & 41.7 & 0.21 & 2.67 & 7.7 & 11.7 \\
\hline 23 & $\mathrm{~S} ? \mathrm{c}$ & 13.31 & 92.7 & 1.91 & 51.3 & 59.5 & 13.4 & 1.33 & 338.5 & 32.4 & 0.23 & 5.69 & 4.0 & 2.6 \\
\hline 24 & $\mathrm{Sc}$ & 13.65 & 90.7 & 1.05 & 27.6 & 41.7 & 8.3 & 2.86 & 135.8 & 45.0 & 0.20 & 3.26 & 6.1 & 10.4 \\
\hline 26 & $\mathrm{Sb}$ & 12.87 & 54.9 & 1.02 & 16.2 & 31.3 & 2.2 & 2.16 & 38.6 & 36.6 & 0.07 & 1.23 & 5.7 & 13.3 \\
\hline 39 & - & 15.40 & 106.0 & 0.66 & 20.3 & 11.4 & 3.7 & 2.33 & 30.6 & 18.6 & 0.32 & 2.68 & 12.1 & 11.5 \\
\hline 63 & - & 15.86 & 93.0 & 0.62 & 16.7 & 5.7 & 7.5 & 7.13 & 111.6 & 102.0 & 1.32 & 19.6 & 6.7 & 42.7 \\
\hline 65 & $\mathrm{SBc}$ & 13.53 & 105.6 & 1.07 & 32.8 & 63.1 & 16.6 & 4.01 & 46.8 & 10.9 & 0.26 & 0.74 & 35.5 & 12.2 \\
\hline 69 & $\mathrm{~S} ? \mathrm{c}$ & 14.54 & 18.6 & 0.89 & 4.8 & 0.8 & 0.3 & 3.24 & 31.7 & 347.9 & 0.39 & 41.2 & 0.95 & 67.5 \\
\hline 98 & - & 13.57 & 64.0 & 0.98 & 18.2 & 22.3 & 3.8 & 3.01 & 77.6 & 59.8 & 0.17 & 3.48 & 4.9 & 16.5 \\
\hline 120 & $\mathrm{SBc}$ & 13.16 & 68.2 & 0.98 & 19.4 & 36.9 & 6.5 & 4.32 & 162.3 & 110.3 & 0.18 & 4.40 & 4.0 & 22.3 \\
\hline 211 & - & 15.15 & 23.4 & 0.81 & 5.5 & 0.7 & 0.8 & 7.21 & 52.9 & 438.8 & 1.14 & 75.60 & 1.5 & 131.1 \\
\hline 216 & - & 15.22 & 106.9 & 0.85 & 26.4 & 13.6 & 5.9 & 2.24 & 63.9 & 23.3 & 0.43 & 4.70 & 9.2 & 8.5 \\
\hline 228 & E-S0 & 14.20 & 131.4 & 1.00 & 38.1 & 52.7 & 5.3 & 0.95 & 334.5 & 58.1 & 0.10 & 6.35 & 1.6 & 2.5 \\
\hline 282 & $\mathrm{Sc}$ & 13.35 & 53.3 & 1.00 & 15.5 & 19.0 & 1.9 & 2.05 & 50.6 & 53.4 & 0.10 & 2.66 & 3.8 & 13.2 \\
\hline 445 & $\mathrm{~S} ? \mathrm{~b}$ & 13.89 & 66.8 & 1.45 & 28.1 & 18.1 & 4.5 & 1.51 & 94.6 & 30.4 & 0.25 & 5.23 & 4.8 & 5.4 \\
\hline 512 & - & 15.56 & 203.1 & 0.16 & 9.4 & 36.0 & 11.7 & 29.30 & 82.2 & 199.2 & 0.33 & 2.28 & 14.2 & 311.7 \\
\hline 544 & $\mathrm{SBbc}$ & 14.24 & 129.2 & 0.91 & 34.1 & 49.1 & 10.2 & 2.29 & 96.4 & 20.9 & 0.21 & 1.96 & 10.6 & 6.7 \\
\hline 546 & $\mathrm{SBc}$ & 13.93 & 98.9 & 1.12 & 32.1 & 38.2 & 12.2 & 3.08 & 84.4 & 20.6 & 0.32 & 2.21 & 14.5 & 9.6 \\
\hline 566 & $\mathrm{Sc}$ & 14.31 & 167.2 & 1.15 & 55.8 & 77.1 & 29.0 & 2.44 & 618.8 & 50.3 & 0.38 & 8.03 & 4.7 & 4.4 \\
\hline 579 & E-S0 & 15.46 & 95.6 & 0.51 & 14.1 & 8.7 & 4.7 & 6.19 & 5.4 & 6.8 & 0.54 & 0.62 & 87.0 & 43.9 \\
\hline
\end{tabular}

Column 1: Number from Kazarian lists.

Column 2: Hubble morphological type taken from LEDA (? means somewhat uncertain type).

Column 3: Corrected integral $B$ magnitudes extracted from LEDA.

Column 4: Distance in Mpc (based on radial velocities reduced to the Local Group and $H=75 \mathrm{~km} \mathrm{~s}^{-1} \mathrm{Mpc}^{-1}$ ).

Column 5: Angular photometric diameter in arcmin taken from LEDA (limiting isophote $B=25^{\mathrm{m}} \operatorname{arcsec}^{-2}$ ).

Column 6: Linear photometric diameter (kpc).

Column \%: Intrinsic $B$ luminosity $\left(10^{9} L_{\odot}\right)$.

Column 8: Mass of neutral hydrogen $\left(10^{9} M_{\odot}\right)$.

Column 9: Neutral hydrogen density projected on the optical disk of the galaxy $\left(10^{-3} \mathrm{~g} \mathrm{~cm}^{-2}\right)$.

Column 10: Indicative total mass $\left(10^{9} M_{\odot}\right)$.

Column 11: Indicative total mass density projected on the optical disk of the galaxy $\left(10^{-3} \mathrm{~g} \mathrm{~cm}^{-2}\right)$.

Column 12: Ratio of neutral hydrogen mass to luminosity $\left(M_{\odot} / L_{\odot}\right)$.

Column 13: Ratio of indicative total mass to luminosity $\left(M_{\odot} / L_{\odot}\right)$.

Column 14: Ratio of neutral hydrogen mass to indicative total mass $(\times 100)$.

Columnq 15: Quasi volumic neutral hydrogen density.

signal-to-noise ratio or signal possibly confused with other sources (see comments on the individual objects given in Paper I).

These data are compared with the corresponding values both for normal and UV-excess galaxies, in order to describe the neutral hydrogen content and its behavior in Kazarian galaxies.

\section{Observational data and derived integral properties}

In order to make valuable comparison with the previous studies, the use of a homogeneous data system is needed.
The increasing number of the available extragalactic $\mathrm{H}$ I data made obvious the necessity to produce a homogenized system which may be used for statistical and comparative purposes. A new homogeneous H I data catalogue has been produced (Bottinelli et al. 1990) and its updated version is now a part of the Lyon-Meudon Extragalactic Database (LEDA), described in detail by Paturel et al. (1997).

It must be noted that all corrections both for optical and H I data were made following the indications given by Paturel et al. (1997) and Bottinelli et al. (1990, 1995). Consequently, all derived (or taken from LEDA) 
integral parameters for Kazarian galaxies correspond to this widely accepted data system.

For distance estimation the radial velocities reduced to the Local Group have been used (adopting $H=75 \mathrm{~km}$ $\left.\mathrm{s}^{-1} \mathrm{Mpc}^{-1}\right)$.

A general description of the calculation method and formulae for all derived parameters may be found in Bottinelli et al. (1982).

In Table 1 the observational data for 23 galaxies taken from our previous article and/or LEDA as well as main derived integral parameters are given.

\section{HI properties of Kazarian galaxies}

In Figs. 1a-d the histograms of the following derived parameters are shown: luminosity $L$, maximum of rotational velocity $V_{\mathrm{m}}$, neutral hydrogen mass $M_{\mathrm{H}}$ and indicative total mass $M_{\mathrm{i}}$. It must be noted that $V_{\mathrm{m}}$ values are available only for 14 galaxies of our sample (see Table 2 in Paper I).

Figure 2 displays the distribution of the relevant ratios $M_{\mathrm{H}} / L, M_{\mathrm{H}} / M_{\mathrm{i}}, M_{\mathrm{i}} / L$ as well as $\sigma_{\mathrm{h}}$, for our sample galaxies. The arrows in Fig. 2 indicate extremal values of the same parameters for classical galaxies which were taken from the earlier works of Balkowski (1973), Shostak (1978), Bottinelli et al. (1982) and more recent data given by Roberts \& Haynes (1994).

It must be noted that with the aim to compare the homogeneous data sets, throughout this paper we tried to use for comparison the data based on observations with Nançay radiotelescope when ever such data were available.

An overview of data given in Table 1 shows that the hydrogen masses in Kazarian galaxies vary in the wide range from $0.310^{9} M_{\odot}$ to $2910^{9} M_{\odot}$ while indicative total masses change from $5.410^{9} M_{\odot}$ to $61910^{9} M_{\odot}$. Compared to this, the ratios $M_{\mathrm{H}} / L, M_{\mathrm{H}} / M_{\mathrm{i}}, M_{\mathrm{i}} / L$ and mean projected $\mathrm{H}$ I density $\sigma_{\mathrm{h}}$ occupy a somewhat smaller range.

Wide dispersion of these values shows clearly that Kazarian galaxies do not constitute a homogeneous sample by none of commonly used H I related parameters.

Distributions presented in Fig. 2 show that the vast majority of our sample galaxies lie within the classical limits, and just a few of them are situated outside.

Data from Table 1 indicate that these galaxies are Kaz 63 and 211 for $M_{\mathrm{H}} / L$ values; $\mathrm{Kaz} 3,63,65,69,211$ and 579 for $M_{\mathrm{i}} / L ; \operatorname{Kaz} 65,69,211$ and 228 for $M_{\mathrm{H}} / M_{\mathrm{i}}$. Finally, Kaz 512 has a peculiar value of $\sigma_{\mathrm{h}}$. They are all listed in Table 2. It must be noted, however that Kaz 3 and 579 cannot be considered with certainty as peculiars due to their $V_{\mathrm{m}}$ and $M_{\mathrm{i}}$ data ambiguity (caused by small inclination) as well as Kaz 512 whose signal is poorly detected with $\mathrm{S} / \mathrm{N}$ ratio less than 3 (see Paper I).

It is well known that neutral hydrogen is rare in elliptical galaxies, and a lack of the meaningful average H I parameters do exist for these systems which in fact contain
Table 2. Peculiar galaxies

\begin{tabular}{lc}
\hline Parameter & Kaz number \\
\hline & \\
\hline$M_{\mathrm{H}} / L$ & 63,211 \\
$M_{\mathrm{H}} / M_{\mathrm{i}}$ & $65,69,211,228$ \\
$M_{\mathrm{i}} / L$ & $3,63,65,69,211,579$ \\
$\sigma_{\mathrm{h}}$ & 512 \\
\hline
\end{tabular}

proportionately lower H I masses. Also, they demonstrate a much larger range in $\mathrm{H} \mathrm{I}$ content and $\sigma_{\mathrm{h}}$ relative to the later spirals (Roberts \& Haynes 1994). In this view, the location of Kaz 228 and 579 outside the usual limits shown in Fig. 2 must be taken with caution.

From the earlier studies it is also known that certain general relationships do exist between $\mathrm{H}$ I related parameters for normal galaxies. We used two well established correlations described by Shostak (1978) for unconfused and non-peculiar objects, namely linear diameter $(\mathbf{A})$ versus corrected $21 \mathrm{~cm}$ line width $\left(\Delta V_{0}\right)$ and $\mathrm{H}$ I mass $\left(M_{\mathrm{H}}\right)$ versus linear diameter plots, in order to check whether or not Kazarian galaxies follow them.

These relationships are:

$\log A=(1.67 \pm 0.39) \log \Delta V_{0}-3.00$

$\log M_{\mathrm{H}}=(1.85 \pm 0.40) \log A+7.22$.

Taking into account the physical significance of the corrected profile width $\Delta V_{0}=\Delta V / \sin i$ and assuming purely circular motions we obtain $\Delta V_{0} \approx 2 V_{\mathrm{m}}$ where $V_{\mathrm{m}}$ is maximum of rotational velocity whose values for 14 Kazarian galaxies are given in Paper I.

In Figs. 3a-b the mean regression lines are shown along with the points representing our sample galaxies.

It is clearly seen that all Kazarian galaxies follow the trend indicated by the mean regression line in Figs. 3a and 3b. However, in Fig. 3a our sample appears to be located above the mean regression line; this is most probably due to the fact that our $V_{\mathrm{m}}$ values were derived by correcting the observed line widths for turbulent motions and thus are, on the mean, smaller than the non-corrected parameter used in Shostak's relation. In Fig. 3b all Kazarian galaxies are dispersed around the mean regression line and only one galaxy (Kaz 512) clearly deviates from that line. In the context of this paper it is more important to demonstrate the location of all Kazarian galaxies (which indicate they generally follow the normal galaxies) than to perform a quantative comparison since our sample galaxies do not comply with of the same restrictions applied by Shostak.

Taking into account all of the aforementioned, the galaxies Kaz 3, 63, 65, 69, 211 and 512 can be considered 

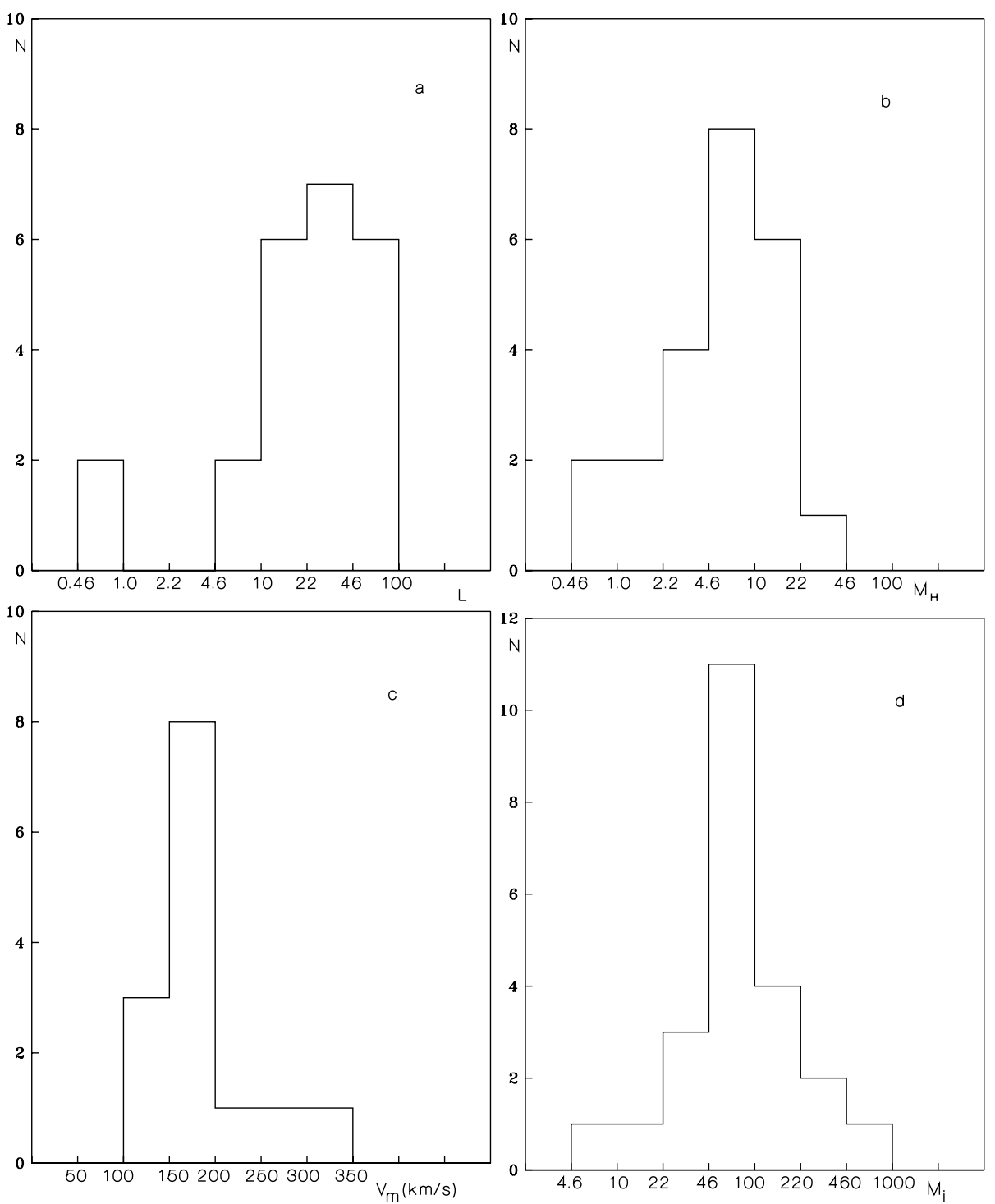

Fig. 1. Distribution of the following parameters for Kazarian galaxies: luminosities, in $10^{9} L_{\odot}$ a); H I masses, in $10^{9} M_{\odot}$ b); maximum of rotational velocities, in $\mathrm{km} \mathrm{s}^{-1} \mathbf{c}$ ); indicative total masses, in $10^{9} M_{\odot} \mathbf{d}$ )

as peculiars possessing $\mathrm{H}$ I related parameters outside the limits representing the classical objects. Some of these objects will be briefly commented on in Sect. 5 .

\section{Comparison with Markarian and blue Haro galaxies}

Comparison with other peculiar galaxies (Markarian and blue Haro, in particular) in the same range of morphological types (Bottinelli et al. 1973a,b; 1975) presented in Table 3 shows that the variation range of derived parameters for Kazarian galaxies is practically the same for $M_{\mathrm{H}} / M_{\mathrm{i}}, M_{\mathrm{i}} / L$ and $\sigma_{\mathrm{h}}$ values. Notice that the units used for comparison are the same as given in Table 1.

In spite of a somewhat different range of the luminosity and hydrogen mass variation compared with Markarians, our sample galaxies vary within the same limits when considering representative ratio $M_{\mathrm{H}} / L$. By this parameter
Kazarian and Markarian galaxies vary in the same and relatively narrow range compared with blue Haro objects.

It is worth noting a well known fact that the same values for classical galaxies (Heidmann 1969; Roberts 1969; Balkowski 1973; Shostak 1978; Bottinelli et al. 1982; Roberts \& Haynes 1994) do not differ from those for Markarians and Haros (Bottinelli et al. 1973a,b; 1975).

The intermixing of Kazarian, Markarian and blue Haro galaxies is well seen on the three-ratio diagram (showing $M_{\mathrm{H}} / M_{\mathrm{i}}$ versus $M_{\mathrm{H}} / L$ plot) displayed in Fig. 4 .

It is well known that primarily a given normal galaxy of the Hubble sequence has particular parameters corresponding to its morphological type (Balkowski 1973; Bottinelli et al. 1982) and then a second approximation these values are also related to the luminosity.

In the case of Kazarian galaxies (as well as Markarians and Haro blue galaxies) not all the integral parameters 

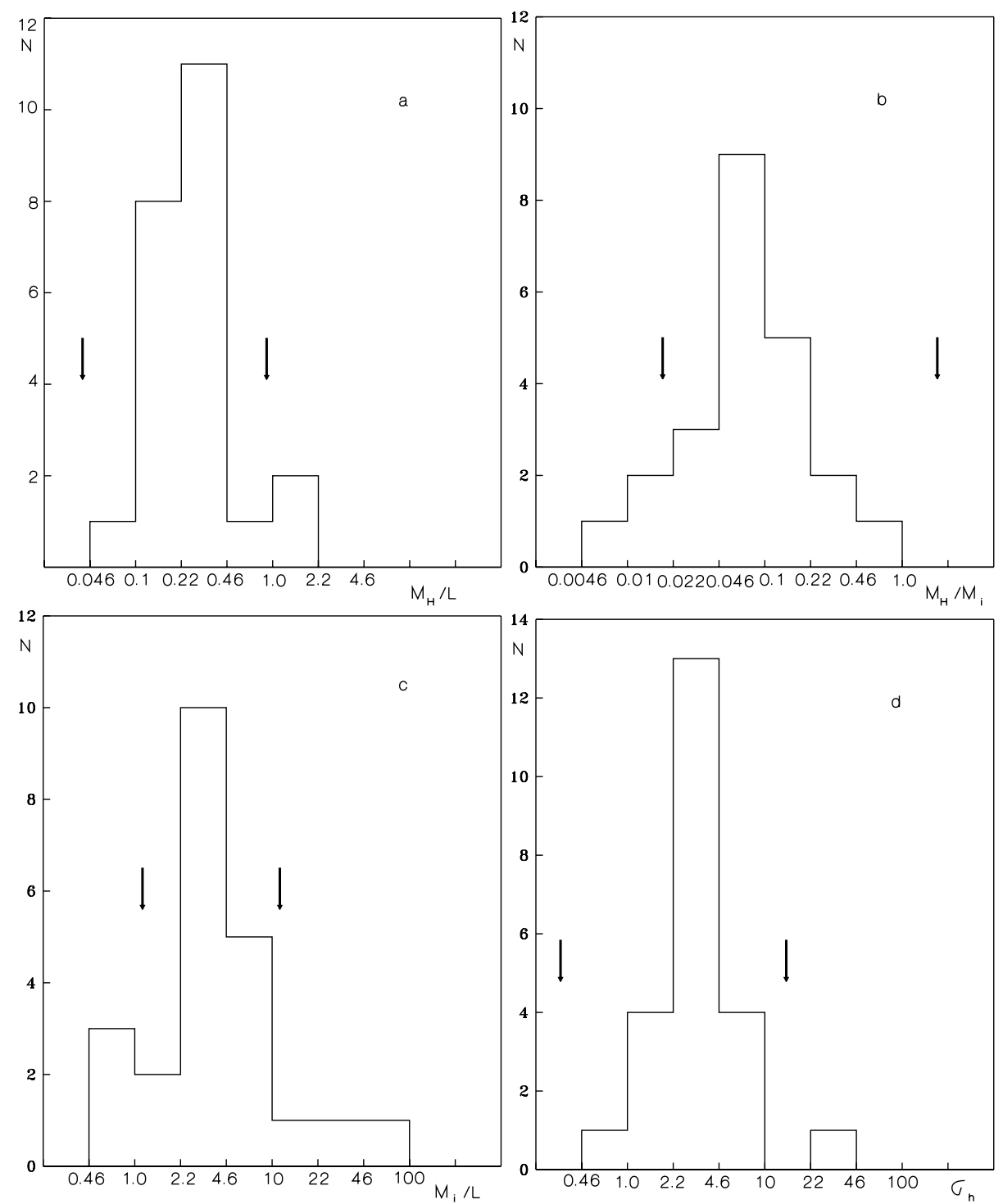

Fig. 2. Distribution of the following parameters for Kazarian galaxies: HI mass to luminosity ratio, in solar units a); H I mass to indicative total mass ratio b); indicative total mass to luminosity ratio, in solar units c); H I mean projected density, in $\left.10^{-3} \mathrm{~g} \mathrm{~cm}^{-2} \mathrm{~d}\right)$

Table 3. H I related parameters variation range

\begin{tabular}{|c|c|c|c|}
\hline \multirow[t]{2}{*}{ Parameter } & \multicolumn{3}{|c|}{ Galaxy type } \\
\hline & Kazarian & Haro & Markarian \\
\hline$M_{\mathrm{H}}$ & $0.30-29.0$ & $0.11-5.4$ & $0.017-11.0$ \\
\hline$L$ & $0.70-77.0$ & $0.3-107.0$ & $0.058-57.0$ \\
\hline$M_{\mathrm{i}}$ & $5.40-619.0$ & $4.4-74.4$ & $1.5-400.0$ \\
\hline$M_{\mathrm{H}} / L$ & $0.07-1.3$ & $0.029-1.1$ & $0.12-3.8$ \\
\hline$M_{\mathrm{H}} / M_{\mathrm{i}}$ & $0.95-87.0$ & $0.92-41.6$ & $1.2-8.8$ \\
\hline$M_{\mathrm{i}} / L$ & $0.62-75.6$ & $0.57-39.5$ & $3.0-62.0$ \\
\hline$\sigma_{\mathrm{h}}$ & $0.95-29.3$ & $0.6-4.1$ & $0.3-7.5$ \\
\hline
\end{tabular}

of a given galaxy have the values expected for a single morphological type. To demonstrate this, we determined for each Kazarian galaxy its most probable morphological type expected from the derived values of the 5 parameters $\left(\sigma_{\mathrm{h}}, \sigma_{\mathrm{h}} / a_{0}, M_{\mathrm{H}} / M_{\mathrm{i}}, M_{\mathrm{H}} / L\right.$ and $\left.W\right)$ following the method described in Bottinelli et al. (1975). Obviously a good agreement with its classical type is always expected when this method is applied for a single galaxy.

As a result, we found only 7 galaxies with all above mentioned parameters corresponding to its given morphological type. A mild agreement was found for 5 galaxies and none for the remaining 11 galaxies of our sample.

Taking into account the luminosity effect which leads to the modification of all used parameters except $\sigma_{\mathrm{h}}$ (Balkowski 1973) and following the scheme described in Bottinelli et al. (1975) we finally found that no classical 

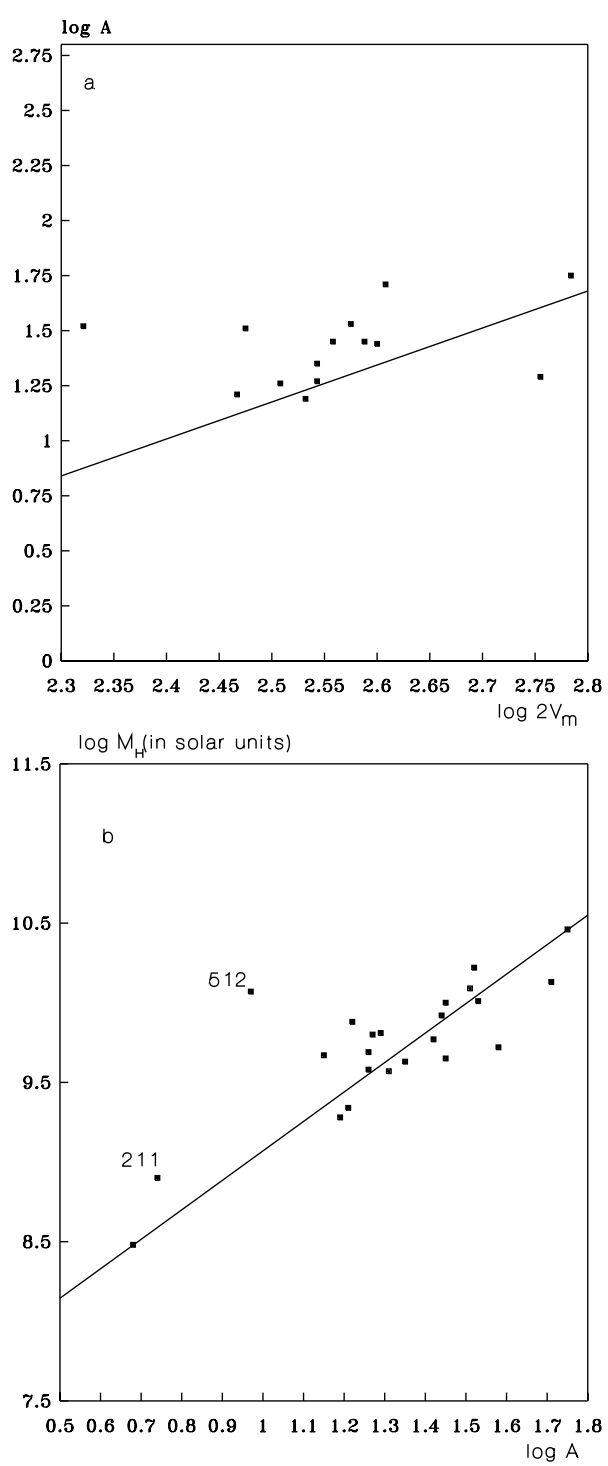

Fig. 3. Linear diameter versus maximum of rotational velocity a) and hydrogen mass versus linear diameter plots b) taken from Shostak (1978). Mean regressions are drawn by solid line (see formulae given in the text)

morphological type can be assigned for 7 galaxies (Kaz 23, $63,69,211,228,512,579)$ while well defined types for 10 galaxies were obtained.

All this suggests a rather high relative number of Kazarian galaxies (7 out of 23) featuring certain peculiarities. The careful comparative study of their optical and H I properties is needed to clarify the nature of these peculiarities.

In the next section brief comments on the optical properties of some peculiar objects are given.

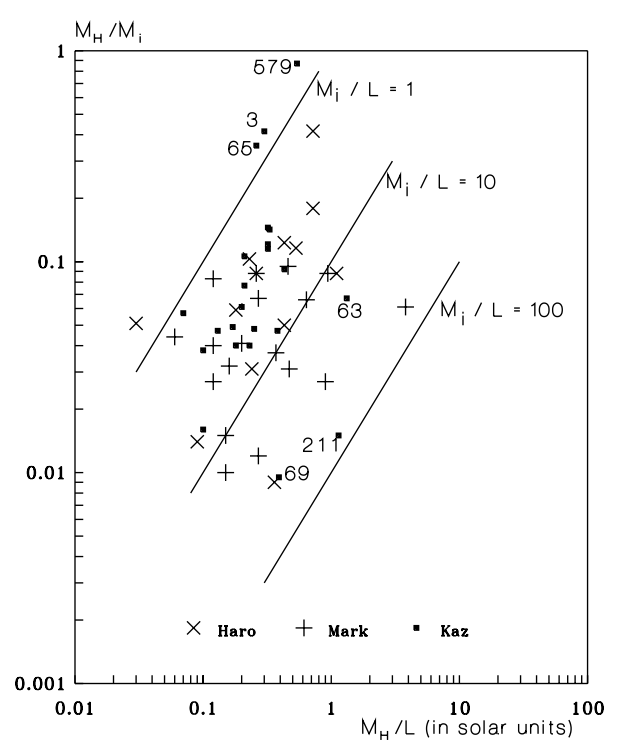

Fig. 4. Three-ratio diagram for the Kazarian, Markarian and blue Haro galaxies displaying H I mass to indicative total mass ratio versus H I mass to luminosity (in solar units). Three values of the total indicative mass to luminosity ratio, in solar units, are also indicated along the straight lines

\section{Brief comments on peculiar galaxies $\mathrm{Kaz} 63, \mathrm{Kaz} 65$, Kaz 69 and Kaz 211}

As it can be seen from Table 2, the galaxies Kaz 63, 65, 69 and 211 have unusual values for at least two $\mathrm{H}$ I related parameters (Kaz 211 is being distinguished by three parameters). These unusual ratios are clear evidence of their peculiar character, so it seems interesting to inquire about their optical data.

Kaz 63 constitutes a pair with UGC 10324 (Paper I). Although its radio signal is rather well separated from that of UGC 10324, the latter may be the cause of such unusual values of $\mathrm{H}$ I mass to luminosity and total mass to luminosity ratios.

The influence of the environment is a well-known phenomenon - for example, the galaxies with close companions have on average a lower HI-to-luminosity ratio than isolated galaxies (Bottinelli 1982). Obviously, Kaz 63 does not follow that trend having (along with Kaz 211) the highest relative H I content among our sample galaxies.

Data on the optical spectra of Kaz 65 (Kazarian 1987) are rather poor: in the range $5600-7000 \mathrm{~A}$ the continuum is faint and the only lines identified are [NII] $6584 \mathrm{~A}$ and $\mathrm{H}_{\alpha}$ which are rather usual for these galaxies.

Kaz 65 (IRAS 16155+6831) was thought to be very luminous X-ray source and included in the Catalogue of cross-correlated sources from ROSAT All-Sky Survey (RASS) with the IRAS Point Source Catalogue published by Boller et al. (1992). More recently, Moran et al. (1994) reported Kaz 65 as a H II galaxy which was removed from the Boller's catalogue by its authors following the new processing of the RASS data. 
Thus, more detailed observational data on Kaz 65 are needed to verify the nature of its peculiar $\mathrm{H}$ I related parameters.

Galaxies Kaz 69 and 211 are studied in detail by Kazarian \& Kazarian (1990). Both are low luminosity dwarfs $\left(M_{\mathrm{pg}}=-16^{\mathrm{m}} 3\right.$ and $-16^{\mathrm{m}} 2$ respectively for Kaz 69 and 211) with rather different physical parameters.

Thus, the electron densities in the central parts of these galaxies are $230 \mathrm{~cm}^{-3}$ and $2800 \mathrm{~cm}^{-3}$ respectively for Kaz 69 and 211 with corresponding total gas amounts of $110^{4} M_{\odot}$ and $2.510^{2} M_{\odot}$ due to the larger size of $\mathrm{Kaz}$ 69.

Full width at half maximum (FWHM) for a number of [O III], [N II], [S II] as well as $\mathrm{H}_{\alpha}$ and $\mathrm{H}_{\beta}$ emission lines in the spectra of $\operatorname{Kaz} 69\left(\approx 300 \mathrm{~km} \mathrm{~s}^{-1}\right)$ are certainly higher than those observed in classical galaxies. There are clear indications that this galaxy is a LINER satisfying criteria given by Heckman (1980).

The elevated value of the indicative total mass for Kaz 69 may be partly caused by a rather high relative amount of the dust evidenced by a narrow dark lane crossing the galaxy buldge, clearly seen on its direct images.

\section{Conclusions}

It is known from the optical data that Kazarian galaxies exhibit large varieties of morphological (Kazarian et al. 1981; Tamazian 1987), spectral (Kazarian et al. 1981; Kazarian \& Tamazian 1982, 1993; Kazarian 1987) and photometric (Tamazian 1983; 1984) properties. From this point of view they are rather close to the galaxies from other peculiar lists such as Markarian (Huchra 1977), blue Haro (du Puy 1970) and Kiso ultraviolet-bright objects (Maehara et al. 1987).

More than $80 \%$ of these galaxies show strong emission lines of Balmer serie and forbidden lines of [O II], [O III], [N II], [S II] etc. frequently observed in the above mentioned lists of UV-excess galaxies which implies significant gas amounts excited by unusually elevated relative number of hot $\mathrm{O}$, B stars in vast majority of these objects.

The present study demonstrate that the same large variety of properties is being observed for non-Seyfert Kazarian galaxies when considering another set of the astrophysical parameters related with their hydrogen content.

We conclude that Kazarian galaxies do not constitute a homogeneous sample by any of the commonly used $\mathrm{H} \mathrm{I}$ related parameters. It is worth noting that these parameters vary in the same range as those for the classical galaxies.

On the other hand, the present study indicates a number of galaxies whose H I properties certainly differ from those for classical normal galaxies, thus showing the peculiar character of a rather high relative number $(\approx 30 \%)$ of Kazarian objects.
Further comparative study of their optical and H I properties would be welcomed to clarify the nature of these peculiarities.

Acknowledgements. The author would like to thank the referee, Prof. L. Bottinelli for valuable comments.

The author has made use of data from Lyon-Meudon Extragalactic Database (LEDA) compiled by the LEDA team at the CRAL-Observatoire de Lyon (France).

\section{References}

Carozzi N., Chamaraux P., Duflot R., 1974, A\&A 30, 21

Balkowski C., 1973, A\&A 29, 43

Boller T., Meurs E.J.A., Brinkmann W., Fink H., Zimmermann U., Adorf H.-M., 1992, A\&A 261, 57

Bottinelli L., Gouguenheim L., Heidmann J., 1973a, A\&A 22, 281

Bottinelli L., Chamaraux P., Gouguenheim L., Heidmann J., 1973b, A\&A 29, 217

Bottinelli L., Duflot R., Gouguenheim L., Heidmann J., 1975, A\&A 41, 61

Bottinelli L., Gouguenheim L., Paturel G., 1982, A\&A 113, 61

Bottinelli L., Gouguenheim L., Fouqué P., Paturel G., 1990, A\&AS 82, 391

Bottinelli L., Gouguenheim L., Paturel G., Teerikorpi P., 1995, A\&A 296, 64

Gouguenheim L., 1969, A\&A 3, 281

Heckman T.M., 1980, A\&A 87, 152

Heidmann N., 1969, Astrophys. Lett. 3, 153

Huchra J.P., 1977, ApJS 35, 171

Kazarian M.A., 1979, Astrofizika 15, 5

Kazarian M.A., 1987, Astrofizika 27, 399

Kazarian M.A., Petrosian A.R., Tamazian V.S., 1981, Sov. Astron. Lett. 7, 648

Kazarian M.A., Kazarian E.S., 1982, Astrofizika 18, 512

Kazarian M.A., Tamazian V.S., 1982, Sov. Astron. Lett. 8, 454

Kazarian M.A., Kazarian E.S., 1990, Astrofizika 33, 169

Maehara H., Noguchi T., Takase B., Handa T., 1987, PASJ 39,1

Maehara H., Hamabe M., Bottinelli L., Gouguenheim L., Heidmann J., Takase B., 1988, PASJ 40, 47

Moran C.E., Halpern J.P., Helfand D.J., 1994, ApJL 433, L65

Paturel G., Andernach H., Bottinelli L., Di Nella H., Durand N., Garnier R., Gouguenheim L., Lanoix P., Marthinet M.C., Petit C., Rouseau J., Theureau G., Vauglin I., 1997, A\&AS 124, 109

Puy D.L. du, 1970, AJ 75, 1143

Roberts M.S., Haynes M.P., 1994, ARA\&A 32, 115

Roberts M.S., 1969, AJ 74, 859

Shostak G.S., 1978, A\&A 68, 321

Solanes J.M., Giovanelli R., Haynes M.P., 1996, ApJ 461, 609

Tamazian V.S., 1983, Soobshch. Byurakan Obs. 56, 80

Tamazian V.S., 1984, Astrofizika 20, 43

Tamazian V.S., 1987, Astrofizika 26, 411

Tamazian V.S., Theureau G., Coudreau N., 1997, A\&AS 126 , 471

Vaucouleurs G. de, Vaucouleurs A. de, Corwin H.G., Buta R.J., Paturel G., Fouque P., 1991, "Third Reference Catalogue of Bright Galaxies" (RC3). Springer, New York 